\title{
中国穿山甲与爪哇穿山甲甲片异速生长分析及其在 司法鉴定中的应用
}

\author{
周昭敏*, 赵 宏, 张忠旭, 王泽晖, 王 晗 \\ (云南云林司法鉴定中心, 云南 昆明 650224)
}

摘要: 甲片对于穿山甲具有重要的防护功能, 其形态差异反映了穿山甲对不同环境的适应性进化。该文对 35 只中国穿山甲和 119 只爪哇穿山甲甲片干重的异速生长模式进行了比较分析。结果表明, 爪哇穿山甲较中国穿山 甲的甲片干重相对于头体长具有更高的异速生长速率 $(P=0.005)$ : 爪哇穿山甲的甲片干重相对于头体长具有正的异 速生长速率 $(b=3.725, P=0.001)$ ，而中国穿山甲的甲片干重相对于头体长则是等速生长 $(b=3.105, P=0.054)$ 。这可能 是由于较低纬度地区生活的爪哇穿山甲较较高纬度地区生活的中国穿山甲受到了更大的捕食压力影响。由于穿山 甲甲片是一种重要传统中药材原料, 相关违法贸易案件频发, 且数量巨大。为推算穿山甲的涉案个体数量, 建议这 两种穿山甲甲片干重与个体数的换算标准为中国穿山甲 $573.47 \mathrm{~g}$, 爪哇穿山甲 $360.51 \mathrm{~g}$, 但是当两种穿山甲大量甲 片混合在一起时, 建议采用上述两个参数的平均值, 即 $466.99 \mathrm{~g}$, 以此为穿山甲甲片贸易案件的司法鉴定以及司 法审判中的定罪量刑提供依据。

关键词: 穿山甲; 甲片; 功能形态; 异速生长; 司法鉴定

中图分类号：Q959.835; Q958.11; DF8 文献标志码：A 文章编号：0254-5853-(2012)03-0271-05

\section{Allometry of scales in Chinese pangolins (Manis pentadactyla) and Malayan pangolins (Manis javanica) and application in judicial expertise}

\author{
ZHOU Zhao-Min ", ZHAO Hong, ZHANG Zhong-Xu, WANG Ze-Hui, WANG Han \\ (Yunnan Yunlin Forensic Center, Kunming 650224, China)
}

\begin{abstract}
Pangolins are unique mammals in that they possess scales that serve a protective biological function. As an important raw material of traditional medicine, illegal trades of these scales are frequent and difficult to investigate or prosecute. We used allometric models of dry weight of scales to compare 35 Chinese pangolins (Manis pentadactyla) and 119 Malayan pangolins (Manis javanica). Our results showed that the dry weight of scales increases significantly faster with the length of head and body in Malayan pangolins $(P=0.005)$, while dry weight of scales is positive (slope $=3.725)$ in Malayan pangolins but isometric (slope=3.105) in Chinese pangolins. The differences in morphology between these species may reflect an evolutionary adaptation to different environments; Malayan pangolins in tropical regions appear to suffer from greater predation pressure than Chinese pangolins in temperate regions. We advise the conversion standards between dry weight of scales and number of individuals as $573.47 \mathrm{~g}$ in Chinese pangolins and $360.51 \mathrm{~g}$ in Malayan pangolins respectively, and when two are mixed together, average above two parameters of the median at $466.99 \mathrm{~g}$. We propose these measurements be used as judicial evidences in forensic identification of related cases.
\end{abstract}

Key words: Pangolin; Scale; Functional morphology; Allometry; Judicial evidence

如果生物的某一形态特征所具有的功能能够 使得物种适合度收益大于损失，则该形态将会维持
或增强; 反之, 这一功能形态则会出现衰退, 因此, 功能形态的差异可以反映出不同物种或种群对各

收稿日期: 2011-12-23; 接受日期: 2012-03-27

基金项目: 云南省森林公安局资助项目

*通信作者(Corresponding author), E-mail: zhouzhaomin@yahoo.cn

第一作者简介: 周昭敏(1981一), 男, 博士, 主要从事野生动物及其制品的司法鉴定,研究领域涉及动物分类学和宏生态学 
自生活环境的适应情况, 例如捕食压力的强弱程度 对防御器官的功能形态具有直接影响(Phillips \& Shine, 2005; Domenici et al, 2008)。

许多作为猎物的物种, 其形态在进化过程中不 断发生改变以降低被捕食风险(Leonard et al, 1999; Nakaoka, 2000; Relyea, 2001)。由于回避被捕食风险 需要付出代价, 许多物种抵御被捕食的能力是随着 被捕食风险的强弱程度而相应地发生改变(Harvell, 1986; Katz \& Dill, 1998; Tollrian \& Harvell, 1999), 因此，在较高捕食压力环境下生活的猎物往往具有 增强的防御功能(Bolser \& Hay, 1996; Menge \& Lubchenco, 1981; Pennings et al, 2001; Vermeij, 1978)。

取食压力呈现出明显的生物地理学模式, 较低 纬度的捕食和植食压力都更强(Bertness et al, 1981; Bolser \& Hay, 1996; Fawcett, 1984; Gaines \& Lubchenco, 1982; Heck \& Wilson, 1987; Jeanne, 1979; Menge \& Lubchenco, 1981; Pennings et al, 2001; Vermeij, 1978)。与较高纬度分布的同属或同 种个体相比，较低纬度分布的被捕食者经受着更强 的捕食压力, 因此, 其具有防御功能的形态在适应 性进化过程中往往会得以增强(Bertness et al, 1981; Vermeij，1978)。穿山甲的甲片具有重要的防御功 能, 一旦来不及逃至避难所身避捕食者, 就会全身 蜷曲，并使甲片直立以阻止捕食者的攻击(Nowak, 1999)。

全世界的穿山甲共有 8 个种(Schlitter, 2005)。 其中，中国穿山甲(Manis pentadactyla)和爪哇穿山 甲 $(M$. javanica) 都只分布在印度马来地区, 但是中 国穿山甲的分布范围较爪哇穿山甲更趋于较高纬 度地区: 中国穿山甲的分布北限在长江一线 $(\sim$ $\left.\mathrm{N} 30^{\circ}\right)$, 南限在泰国北部和缅甸 $\left(\sim \mathrm{S} 20^{\circ}\right)$; 而爪哇穿 山甲的分布则北限在中国云南 $\left(\sim \mathrm{N} 25^{\circ}\right)$, 南限在婆 罗洲一带 $\left(\sim \mathrm{S} 10^{\circ}\right)$ (Nowak, 1999; Wu et al, 2005)。中 国穿山甲和爪哇穿山甲分布区有交叠, 但存在明显 的纬度差异,前者分布区主要在温带; 而后者的分布 区主要在热带。本文拟对中国穿山甲和爪哇穿山甲 的甲片干重进行异速生长分析, 以揭示它们在不同 捕食压力下的适应性进化。

穿山甲被列为我国 II 级重点保护陆生野生动 物和 CITES 附录-II物种(CITES, 2010), 其甲片的 贸易是被法律明文禁止的。《最高人民法院关于审 理破坏野生动物资源刑事案件具体应用法律若干
问题的解释》(法释[2000]37 号文)关于非法猎捕、 杀害、收购、运输, 以及出售珍贵、濒危野生动物 刑事案件“情节严重”、“情节特别严重”的数量认定 标准, 规定了以涉案动物个体数量和价值标准的多 少为定罪量刑依据。涉案动物制品的价值标准认定 则是基于个体数量乘以相应物种的法定资源管理 费, 因此, 涉案动物个体数量的认定是定罪量刑的 必要条件。作为一种重要的传统中药材原料, 穿山 甲甲片的贸易案件频发, 且数量巨大。以云南省森 林公安局 “四一六” 和 “一一・五” 特大穿山甲 甲片走私案为例, 这两个案子共查获约 $1.09 \mathrm{t}$ 甲片。 然而, 由于缺乏科学的转换标准, 难以通过所查获 穿山甲甲片的重量推测涉案的个体数量, 进而确定 其价值标准，因此，在异速生长分析的基础之上, 本文将利用大量样本统计出中国穿山甲和爪哇穿 山甲甲片干重与个体数的详细转换标准, 为相关个 体数的推测提供科学依据。

\section{1 材料与方法}

\section{1 实验动物}

2009 年, 云南省森林公安局在办理案件中查获 了 154 只穿山甲(死体), 冰冻保存于云南省野生动 物园。该批穿山甲包括 35 只中国穿山甲(15万和 20) 和 119 只爪哇穿山甲(58ふ和 61 웅。

\section{2 数据收集}

用卷尺测量各个个体的头体长(length of head and body, LHB)、尾长(tail length)、后足长(length of hind feet, LHF)等 3 个指标, 并对该批穿山甲进行水 煮、剥片、晒干。为了尽量降低甲片含水量, 从每 个物种各选取 5 个个体的甲片作为样本, 每隔一天 监测一次, 直至样本干重趋于稳定, 至此, 甲片露 天晾晒时间共计 $45 \mathrm{~d}$ 。用电子秤(量程 $2000 \mathrm{~g}$, 分度 值 $0.01 \mathrm{~g}$ )测定各个体的甲片干重。

\section{3 数据处理}

异速生长分析的公式为 $Y=\mathrm{a} X^{b}$, 其中 $X$ 为自变 量, $Y$ 为因变量, $\mathrm{a}$ 为异速生长常数, $b$ 为异速生长指 数(Peters，1983)。头体长、尾长和后足长分别被定 义为自变量 $X$, 甲片干重则作为因变量。自变量为 一维变量, 而因变量为三维变量, 因此, 当 $b=3.00$ 时, $Y$ 与 $X$ 间为等速生长; 当 $b>3.00$ 或 $b<3.00$ 时, $Y$ 对 $X$ 分别为正或负的异速生长。各变量经过常用 对数转换后, 应用 RMA 软件(Bohonak, 2004)进行 压轴回归分析(reduced major axis regression), 压轴 
回归分析比最小二乘回归分析 (least squares regressions)更适合应用于评价具有明显误差离散的 两组变量的结构关系(Rayner, 1985)。其余统计则应 用 SPSS17.0 软件进行分析。此外，还统计了中国穿 山甲和爪哇穿山甲单个个体甲片干重的算术平均 值、极值和中位数。

\section{2 结 果}

\section{1 异速生长}

异速生长分析结果详见表 1、2。中国穿山甲的 甲片干重与自变量的异速生长指数无显著的性别
差异(头体长: $P=0.101$; 尾长: $P=0.454$; 后足长: $P=0.937)$; 而爪哇穿山甲的甲片干重与头体长的相 对生长速率无显著的性别差异 $(P=0.088)$, 但与尾长 $(P=0.034)$ 和后足长 $(P=0.001)$ 的异速生长指数有显 著的性别差异。同时，两种穿山甲的甲片干重与头 体长均有较高的相关系数, 于是我们选取头体长作 为代表体形大小的指标。爪哇穿山甲与中国穿山甲 甲片干重的异速生长指数存在显著性差异 $(P=0.005)$ 。中国穿山甲的甲片干重与头体长呈等速 生长 $(b=3.105, P=0.054)$; 而爪哇穿山甲的甲片干重 与头体长呈正的异速生长 $(b=3.725, P=0.001)$ 。

表 1 中国穿山甲甲片干重的压轴回归分析结果

Tab. 1 Summary of reduced major axis regression statistics for dry weight of scales in Chinese pangolins

\begin{tabular}{|c|c|c|c|c|c|c|}
\hline \multirow{2}{*}{$\begin{array}{c}\text { 变量 } \\
\text { Variables }\end{array}$} & \multicolumn{2}{|c|}{ 雄 Male $(n=15)$} & \multicolumn{2}{|c|}{ 雌 Female $(n=20)$} & \multicolumn{2}{|c|}{ 雄+雌 Male and female $(n=35)$} \\
\hline & $R^{2}$ & 斜率 Slope $(S E)$ & $R^{2}$ & 斜率 Slope $(S E)$ & $R^{2}$ & 斜率 Slope (SE) \\
\hline $\begin{array}{c}\text { 头体长 } \\
\text { LHB }\end{array}$ & 0.807 & $3.364(0.410)$ & 0.342 & $3.005(0.342)$ & 0.547 & $3.105(0.364)$ \\
\hline $\begin{array}{c}\text { 尾长 } \\
\text { TL }\end{array}$ & 0.738 & $3.173(0.450)$ & 0.600 & $2.896(0.432)$ & 0.667 & $3.004(0.302)$ \\
\hline $\begin{array}{c}\text { 后足长 } \\
\text { LHF } \\
\end{array}$ & 0.712 & $3.997(0.595)$ & 0.309 & $6.605(1.294)$ & 0.524 & $4.772(0.573)$ \\
\hline
\end{tabular}

表 2 爪哇穿山甲甲片干重的压轴回归分析结果

Tab. 2 Summary of reduced major axis regression statistics for dry weight of scales in Malayan pangolins

\begin{tabular}{|c|c|c|c|c|c|c|}
\hline \multirow{2}{*}{$\begin{array}{c}\text { 变量 } \\
\text { Variables }\end{array}$} & \multicolumn{2}{|c|}{ 雄 Male $(n=58)$} & \multicolumn{2}{|c|}{ 雌 Female $(n=61)$} & \multicolumn{2}{|c|}{ 雄+雌 Male and female $(n=119)$} \\
\hline & $R^{2}$ & 斜率 Slope (SE) & $R^{2}$ & 斜率 Slope (SE) & $R^{2}$ & 斜率 Slope $(S E)$ \\
\hline $\begin{array}{c}\text { 头体长 } \\
\text { LHB }\end{array}$ & 0.918 & $3.884(0.148)$ & 0.763 & $3.697(0.234)$ & 0.861 & $3.725(0.128)$ \\
\hline $\begin{array}{c}\text { 尾长 } \\
\text { TL }\end{array}$ & 0.883 & $3.067(0.140)$ & 0.599 & $2.981(0.246)$ & 0.794 & $2.986(0.125)$ \\
\hline $\begin{array}{c}\text { 后足长 } \\
\text { LHF }\end{array}$ & 0.545 & $5.546(0.500)$ & 0.112 & $4.426(0.543)$ & 0.349 & $4.577(0.341)$ \\
\hline
\end{tabular}

\section{2 个体数推算}

154 只穿山甲的平均甲片干重为 (423.47 \pm 197.19$)$ g (27.19 1121.07 g)。35 只中国穿山 甲与 119 只爪哇穿山甲在单个个体甲片干重存在显 著性差异 $(P<0.001)$, 并且上述异速生长分析结果也 表明，即使考虑到个体体形大小对甲片干重的影响， 两者的甲片干重依然存在显著性差异, 因此, 有必 要分别计算两种穿山甲的参数标准。35只中国穿山 甲的平均甲片干重为 $(592.99 \pm 217.63) \mathrm{g}$ $(129.47 \sim 1121.07 \mathrm{~g})$, 中位数为 $573.47 \mathrm{~g}$ 。119 只爪哇 穿山甲的平均甲片干重为 $(373.61 \pm 160.43) \mathrm{g}$ $(27.19 \sim 824.54 \mathrm{~g})$, 中位数为 $360.51 \mathrm{~g}$ 。

\section{3 讨 论}

猎物抵御被捕食风险的策略是多种多样的, 例 如改变功能形态(Leonard et al, 1999; Nakaoka, 2000;
Vermeij, 1978)、提高化学防御水平(Bolser \& Hay, 1996; Pennings et al, 2001)以及行为方式和生境选 择的转变(Bertness et al, 1981; Fawcett, 1984; Martin, 2000; Turner \& Mittlebach, 1990)。本文证实了中国 穿山甲和爪哇穿山甲这两个近缘物种在防御形态 上的差异: 中国穿山甲甲片干重相对于头体长是等 速生长, 而爪哇穿山甲则呈正的异速生长。

捕食风险的强弱是与纬度高低有关的。高纬度 地区的捕食压力较低已得到广泛的证明，例如鸟类 的巢捕食风险就随着纬度梯度增高而降低 (McKinnon, 2010), 因此, 较低纬度分布的爪哇穿山 甲比较高纬度分布的中国穿山甲承受着更高的捕 食压力。相应地, 为了增强防御功能, 爪哇穿山甲 的个体甲片干重为正的异速生长速率, 以实现其在 高捕食压力下的适应性进化。

中国穿山甲与爪哇穿山甲在个体甲片干重生长 
速率上的差异还提供了穿山甲这个支系在起源初 期所处自然环境的信息。中国穿山甲的分布区主要 在温带地区, 其个体甲片干重与体形大小呈等比例 增加, 表明该物种为甲片发育所付出的代价处于理 想的平衡状态; 而在较低纬度地区生活的爪哇穿山 甲为了抵御更高的捕食风险，其个体甲片干重与体 形大小呈正比例增加, 即为甲片的发育付出了更高 的代价。一个成功的入侵物种往往会通过功能形态 的适应性改变而适应新的生活环境 (Phillips \& Shine, 2005), 因此, 穿山甲可能起源于温带地区, 而爪哇穿山甲的祖先是后来迁入具有较高捕食压 力的热带地区，同时爪哇穿山甲的个体甲片干重生 长速率作为可塑的表型特征发生了相应的适应性 进化, 使得具有防御功能的甲片在形态上得以增 强。发现于德国麦塞尔化石遗址(Messel fossil deposit)的始新世中期 Eomanis 化石是目前已知最 古老的穿山甲化石(Franzen, 2005; Meredith et al, 2009), 这也佐证了我们关于穿山甲这一支系从高 纬度地区向低纬度地区扩张的推测。

虽然国内还进行过穿山甲一些生理(Chen et al, 1991)和食物(Ke et al, 1999)方面的研究, 但目前人 们对其认识还是非常有限。在今后对穿山甲研究工 作中, 有两个方向是值得注意的: 一是通过行为研 究对比分析不同纬度分布的各穿山甲物种所承受 的捕食压力差异; 二是尽量将更多的物种纳入分 析，毕竟全世界只有 8 种穿山甲。

由于在司法实践中涉案甲片往往数量巨大,
且受穿山甲个体大小差异和不同部位甲片形态 差异等因素的影响, 因此, 无法精确推算涉案甲 片所对应的穿山甲个体数, 只能采用某一较为 合理的参数进行换算以满足司法需求。中位数 与数据的排列位置有关, 是一组数据中间位置 上的代表值, 不受数据极端值的影响, 因此, 极 端数据的变动对中位数影响不大。相对于算术平 均值和极值, 中位数更适宜作为利用穿山甲甲片重 量推测其个体数的标准参数。根据《司法鉴定程序 通则》第二十二条之规定，司法鉴定应当依次遵循 国家标准和技术规范，司法鉴定主管部门、司法鉴 定行业组织或者相关行业主管部门制定的行业标 准有和技术规范, 该专业领域多数专家认可的技术 标准和技术规范，最后是可以采用所属司法鉴定机 构自行制定的有关技术规范，因此，在没有现成的 标准和技术规范的情况下，我们建议在司法鉴定实 践中利用穿山甲甲片干重推算其个体数时，具体转 换参数为：中国穿山甲为 $573.47 \mathrm{~g}$; 爪哇穿山甲为 $360.51 \mathrm{~g}$ 。中国穿山甲甲片甲片较厚，且背甲远端针 圆，而爪哇穿山甲甲片较薄，且背甲远端呈凸突 状; 但是当两种穿山甲大量甲片混合在一起, 为了 提高司法鉴定效率, 以满足司法程序对时效的要求, 建议采用上述两个参数的平均值, 即 $466.99 \mathrm{~g}$ 。

致谢: 感谢中国科学院昆明动物研究所王应祥 研究员、蒋学龙研究员和西南林业大学刘宁教授在 数据分析上给予的指导, 以及云南野生动物园王建 军先生在数据采集过程中提供的大力帮助。

\section{参考文献:}

Bertness MD, Garrity SD, Levings SC. 1981. Predation pressure and gastropod foraging: a tropical-temperate comparison[J]. Evolution, 35(5): 995-1007.

Bohonak AJ. 2004. RMA, Version 1.17: Software for Reduced Major Axis Regression[M]. San Diego: San Diego State University.

Bolser RC, Hay ME. 1996. Are tropical plants better defended? Palatability and defenses of temperate vs. tropical seaweeds[J]. Ecology, 77(8): 2269-2286

Chen Q, Liu RQ, Wang YX, Shi LM. 1991. Studies on the mitotic chromosomes and meiotic synaptonemal complexes (SC) of Chinese pangolin (Manis pentadactyla) [J]. Zool Res, 12(3): 299-304. [陈全, 刘 瑞清, 王应祥, 施立明. 1991. 中国穿山甲有丝分裂染色体和减数 分裂联会复合体(SC)的研究. 动物学研究, 12(3): 299-304.]

Domenici P, Turesson H, Brodersen J, Brönmark C. 2008. Predator-induced morphology enhances escape locomotion in crucian carp $[\mathrm{J}]$. Proc $R$ Soc B: Biol Sci, 275(1631): 195-201.

Fawcett MH. 1984. Local and latitudinal variation in predation on an herbivorous marine snail[J]. Ecology, 65(4): 1214-1230.

Franzen JL. 2005. The implications of the numerical dating of the Messel fossil deposit (Eocene, Germany) for mammalian biochronology[J]. Ann Paléontol, 91(4): 329-335.

Gaines SD, Lubchenco J. 1982. A unified approach to marine plant-herbivore interactions. II. Biogeography[J]. Annu Rev Ecol Syst, 13(1): 111-138.

Harvell CD. 1986. The ecology and evolution of inducible defenses in a marine bryozoan: cues, costs, and consequences[J]. Am Nat, 128(6): 810-823.

Heck KL Jr, Wilson KA. 1987. Predation rates on decapod crustaceans in latitudinally separated seagrass communities: a study of spatial and temporal variation using tethering techniques[J]. J Exp Mar Biol Ecol, 107(2): 87-100.

Jeanne RL. 1979. A latitudinal gradient in rates of ant predation[J]. Ecology, 60(6): 1211-1224.

Katz LB, Dill LM. 1998. The scent of death: chemosensory assessment of 
predation risk by prey animals[J]. Ecoscience, 5(3): 361-394.

Ke YY, Chang H, Wu SB, Liu Q, Feng GX. 1999. A study of Chinese pangolin's main food nutrition[J]. Zool Res, 20(5): 394-395. [柯亚永, 常弘, 吴诗宝, 刘茜, 冯干新. 1999. 穿山甲主要食物营养成分研究. 动物学研究, 20(5): 394-395.]

Leonard GH, Bertness MD, Yund PO. 1999. Crab predation, waterborne cues, and inducible defenses in the blue mussel, Mytilus edulis[J]. Ecology, 80(1): 1-14.

Martin TE, Martin PR, Olson CR, Heidinger BJ, Fontaine JJ. 2000. Parental care and clutch sizes in North and South American birds[J]. Science, 287(5457): 1482-1485.

McKinnon L , Smith PA, Nol E, Martin JL, Doyle FI, Abraham KF, Gilchrist HG, Morrison RIG, Bêty J. 2010. Lower predation risk for migratory birds at high latitudes[J]. Science, 327(5963): 326-327.

Menge BA, Lubchenco J. 1981. Community organization in temperate and tropical rocky intertidal habitats: prey refuges in relation to consumer pressure gradients[J]. Ecol Monogr, 51(4): 429-450.

Meredith RW, Gatesy J, Murphy WJ, Ryder OA, Springer MS. 2009. Molecular decay of the tooth gene Enamelin (ENAM) mirrors the loss of enamel in the fossil record of placental mammals[J]. PLoS Genet, 5(9): e1000634.

Nakaoka M. 2000. Nonlethal effects of predators on prey populations: predator-mediated change in bivalve growth[J]. Ecology, 81(4): 1031-1045.

Nowak RM. 1999. Walker's Mammals of the World[M]. 6th ed. Baltimore: The Johns Hopkins University Press.

Pennings SC, Siska EL, Bertness MD. 2001. Latitudinal differences in plant palatability in Atlantic Coast salt marshes[J]. Ecology, 82(5): 1344-1359.

Peters RH. 1983. The Ecological Implications of Body Size[M]. Cambridge: Cambridge University Press.

Phillips BL, Shine R. 2005. The morphology, and hence impact, of an invasive species (the cane toad, Bufo marinus): changes with time since colonisation[J]. Anim Conserv, 8(4): 407-413.

Rayner JMV. 1985. Linear relations in biomechanics: the statistics of scaling functions[J]. J Zool: A, 206(3): 415-439.

Relyea RA. 2001. Morphological and behavioral plasticity of larval anurans in response to different predators[J]. Ecology, 82(2): 523-540.

Schlitter DA. 2005. Order pholidota[M] // Wilson DE, Reeder DM. Mammal Species of the World: A Taxonomic and Geographic Reference. 3rd ed. Baltimore: Johns Hopkins University Press, 530-531.

Tollrian RI, Harvell CD. 1999. The Ecology and Evolution of Inducible Defenses[M]. Princeton: Princeton University Press.

Turner AM, Mittlebach GG. 1990. Predator avoidance and community structure: interactions among piscivores, planktivores, and plankton[J]. Ecology, 71(6): 2241-2254.

Vermeij GJ. 1978. Biogeography and Adaptation[M]. Cambridge: Harvard University Press.

Wu SB, Wang YX, Feng Q. 2005. A new record of Mammalia in China-Manis Javanica[J]. Acta Zootaxon Sin, 30(2): 440-443. [吴诗宝, 王应祥, 冯庆. 2005. 中国兽类一新纪录一一爪哇穿山甲. 动物分 类学报, 30(2): 440-443.] 